\section{BRAZIULIAN JOURNAL}

OF MEDICAL AND BIOLOGICAL RESFARCH

www.bjournal.com.br
ISSN 0100-879X

Volume 43 (12) 1135-1244 December 2010

BIOMEDICAL SCIENCES

AND

CLINICAL INVESTIGATION

Braz J Med Biol Res, December 2010, Volume 43(12) 1232-1238

doi: 10.1590/S0100-879X2010007500133

The efficacy of YKL-40 and CA125 as biomarkers for epithelial ovarian cancer

L. Zou, X. He and J.W. Zhang

The Brazilian Journal of Medical and Biological Research is partially financed by
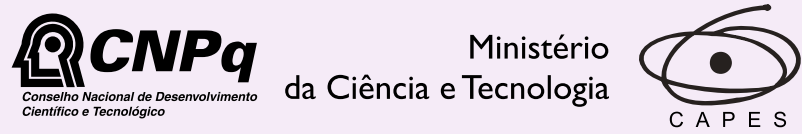

Ministério da Educação

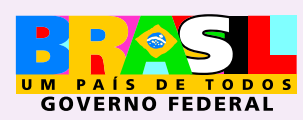

DFAPESP

Institutional Sponsors
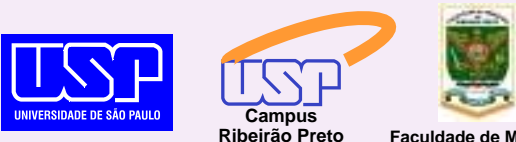

$\oplus$ SHIMADZU

GE Healthcare
Hotsite of proteomics metabolomics developped by: 


\title{
The efficacy of YKL-40 and CA125 as biomarkers for epithelial ovarian cancer
}

\author{
L. Zou, X. He and J.W. Zhang \\ Department of Gynecology, Second West China Hospital, Sichuan University, Chengdu, Sichuan, China
}

\begin{abstract}
Our objective was to estimate the efficacy of the measurement of serum YKL-40 alone or with CA125 as biomarkers for the diagnosis of epithelial ovarian cancer (EOC) using the YKL-40 ELISA kit. An experimental group of 49 ovarian cancer patients included 42 patients with EOC (53 \pm 15 years, range: $19-81$ years) and 7 patients (48 \pm 13 years, range: 29-36 years) with borderline epithelial ovarian tumor. A control group of 88 non-malignant cases included 42 patients (43 \pm 10 years, range: $26-$ 77 years) with benign gynecological disease and 46 healthy women ( $45 \pm 14$ years, range: $30-68$ years) at a teaching hospital. Both YKL-40 (220.1 \pm 94.1 vs $61.6 \pm 48.4$ and $50.1 \pm 41.2 \mathrm{ng} / \mathrm{mL})$ and CA125 (524.9 \pm 972.5 vs $13.4 \pm 7.6$ and $28.5 \pm 29.6$ $\mathrm{U} / \mathrm{mL})$ levels were significantly higher $(P<0.05)$ in patients with ovarian cancer compared to the healthy and non-malignant groups. YKL-40 had $92.9 \%$ sensitivity and $94.4 \%$ specificity for the diagnosis of EOC. When YKL-40 and CA125 were tested in parallel, the sensitivity was increased to $98.2 \%$, but the specificity was decreased to $81.3 \%$. The correlations between serum YKL-40 and tumor stage, grade histology, performance status, patient age, and extension of debulking surgery were tested. With increasing stage and grade of EOC, preoperative serum YKL-40 levels were significantly increased $(P=0.029, P=0.05$, respectively). Serum YKL-40 alone or with serum CA125 levels are useful, although with some limitations, to diagnose ovarian cancer. Our study showed that YKL-40 may not be an independent prognostic factor for ovarian cancer. This prospective study may be a new trend in looking for biomarkers that optimize diagnosis of ovarian cancer.
\end{abstract}

Key words: YKL-40; CA125; Ovarian cancer; Diagnosis

\section{Introduction}

YKL-40, a secreted glycoprotein belonging to the chitinase protein family (1), was identified in a search for new bone proteins in 1992. The name "YKL-40" was based on its three $\mathrm{NH}_{2}$-terminal amino acids tyrosine $(\mathrm{Y})$, lysine $(\mathrm{K})$, and leucine $(\mathrm{L})$ and its molecular weight of $40 \mathrm{kDa}$. The gene of YKL-40 is located on chromosome 1q32 (2).

YKL-40 is secreted by a variety of human nonmalignant cells, including monocytes, macrophages, articular cartilage cells, and synovial cells. Studies have indicated that YKL-40 levels were elevated in patients with inflammation $(3,4)$. Nevertheless, YKL-40 was mainly secreted by tumor-associated macrophages, monocytes, neutrophils, and tumor cells (5). A number of studies have suggested that YKL-40 was highly expressed in many solid tumors, such as primary colorectal cancer (6), metastatic breast cancer (7), cervical cancer (8), endometrial carcinoma (9), recurrent ovarian cancer (10), and epithelial ovarian cancer (EOC) (11).

Ovarian cancer is the most common cause of cancer death associated with gynecological tumors. More than $70 \%$ of patients with EOC are diagnosed with advanced stage and the 5-year survival rate is approximately $30 \%$ among these patients (12). Cure rate for early stage (I-II) EOC is approximately 70 to $90 \%$ (13). Thus, the early detection of EOC is essential to improve survival rates. Until now, there is no efficient method for the preoperative diagnosis of ovarian cancer. CA125 is the most widely used marker for EOC, especially for monitoring the response to treatment and recurrence of malignant disease. However, using CA125 alone is not an ideal method for preoperative diagnosis. Serum CA125 has been reported to diagnose advanced-stage EOC with 87 to $94 \%$ sensitivity. However, the sensitivity of diagnosing stage I was as low as 27 to $66 \%$ (14).

The search for new tumor markers or biomarkers to improve the sensitivity of EOC diagnosis is a challenge. A retrospective clinical study of patients with different types of solid tumors indicated that the serum level of $Y K L-40$ was elevated in patients compared to healthy subjects and the

Correspondence: J.W. Zhang, Department of Gynecology, Second West China Hospital, Sichuan University, N0 20, Section 3, South People's Road, Chengdu, Sichuan 610041, China. Fax: +86-28-8555-9065. E-mail: zhangjw163@163.com

Received April 27, 2010. Accepted November 3, 2010. Available online November 26, 2010. Published December 20, 2010. 
highest serum YKL-40 levels were detected in patients with advanced cancer and with the poorest prognosis (15). It has been suggested that $Y K L-40$ is a better biomarker for the diagnosis of early-stage EOC than CA125 and CA15-3 (11). High YKL-40 expression has been reported in cancer cells and in preoperative blood from patients with borderline tumors and EOC (11).

In the present study, we determined the serum values of YKL-40 and CA125 and estimated the efficacy of the measurement of YKL-40 with or without CA125 for the diagnosis of EOC, and also investigated the prognostic role of $\mathrm{YKL}-40$ in $\mathrm{EOC}$.

\section{Material and Methods}

From May 2007 to January 2008, patients undergoing gynecologic-oncologic surgeries in the Gynecology Department of Second West China Hospital, Sichuan University, had their preoperative serum samples banked after signing informed consents. The group with malignancies, based on the pathologic diagnosis after surgery, included 42 patients with EOC and 7 patients with borderline epithelial ovarian tumor. The control group included two subgroups, 42 patients with benign gynecological diseases and 46 healthy women. Any women with conditions that could influence YKL-40 levels, such as arthritis or any other kind of cancer, were excluded. The collection and use of all blood samples were approved by the Ethics Committee of Sichuan University and were in accordance with all regulations and guidelines of China. After being informed about the details of the research, patients gave written informed consent 1-2 weeks before surgery and healthy women did so during routine examination. A $5-\mathrm{mL}$ blood sample was collected from each subject, clotted at room temperature for about $1 \mathrm{~h}$, and centrifuged at $4^{\circ} \mathrm{C}$ for $5 \mathrm{~min}$ at 1500 $\mathrm{rpm}$. The aliquot of serum was then subdivided into small volumes and stored at $-80^{\circ} \mathrm{C}$.

YKL-40 levels were determined in all serum samples using the YKL-40 enzyme-linked immunosorbent assay (ELISA) kit (Quidel Corporation, USA) according to manufacturer instructions. YKL-40 serum concentrations were determined by measurement of absorbance at $450 \mathrm{~nm}$, which was read against a standard curve. The receiver operating characteristics (ROC) curves of YKL-40 and CA125 were constructed for EOC patients vs controls. The cut-off level of YKL-40 was determined according to ROC curves.

All serum CA125 levels were determined by an experienced researcher. The value of $35 \mathrm{U} / \mathrm{mL}$ was taken as a the threshold of CA125 for predicting the possible diagnosis of ovarian cancer.

All patients with EOC were followed in the outpatient department of our hospital after surgery. Their information, including performance status, recurrence and chemotherapy after surgery, was recorded and their date of death was confirmed by the families.

\section{Statistical analysis}

Patients were divided into two subgroups in both the malignant and control groups according to their clinical and pathological parameters. The least-significant difference (LSD) test was used for multiple comparisons of the difference in serum YKL-40 and CA125 between subgroups. Correlations between serum YKL-40 and International Federation of Gynecology and Obstetrics (FIGO) stage, between YKL-40 and pathological grade, and between YKL-40 and histology were analyzed by the Spearman rank-correlation coefficient. The prognostic value of YKL-40 was assessed by univariate Cox analysis and multivariate Cox analysis, including serum YKL-40, serum CA125, FIGO stage, and histological grade, respectively. Kaplan-Meier survival estimates were used to determine the different survival rates of patients grouped by normal or elevated plasma YKL-40 levels. The Statistical Package for the Social Sciences Version 13.0 software (SPSS Inc., USA) was used for all analyses and the difference was considered to be significant at $\mathrm{P} \leq 0.05$.

\section{Results}

The characteristics of 42 patients with EOC are listed in Table 1. Fourteen (33.3\%) patients were in the early stage (FIGO stage I-II) while the rest were in the advanced stage (FIGO stage III-IV). Thirty-one $(73.8 \%)$ cancers were histological grade 3 . Serous and non-serous histology was

Table 1. Demographic characteristics of patients with epithelial ovarian cancer.

\begin{tabular}{lc}
\hline Characteristics & Number (\%) \\
\hline FIGO stage & \\
I & $11(26.1 \%)$ \\
III & $3(7.2 \%)$ \\
IV & $27(64.3 \%)$ \\
Grade & $1(2.4 \%)$ \\
I & \\
2 & $3(7.1 \%)$ \\
3 & $8(19.1 \%)$ \\
Histology & $31(73.8 \%)$ \\
Serous & \\
Endometrioid & $18(42.9 \%)$ \\
Clear cell & $3(7.1 \%)$ \\
Mixed gland & $3(7.1 \%)$ \\
Other $^{+}$ & $14(33.3 \%)$ \\
\hline
\end{tabular}

The mean age of the patients was 53 years (range 19-81 years). FIGO = International Federation of Gynecology and Obstetrics. *Including squamous, small cell, and carcinosarcoma. ${ }^{+}$Including gland plus squamous, endometrioid plus clear cell, serous plus clear cell, and unclassified gland. 
available for $42.9 \%(18 / 42)$ and $57.1 \%$ (24/42) patients, respectively (Table 1$)$.

The preoperative serum levels of YKL-40 and CA125 in each subgroup are listed in Table 2 . There was no statistically significant difference $(P>0.05)$ between subgroups in the nonmalignant or malignant group. When each subgroup in the malignant group was compared to each subgroup in the nonmalignant group, both $\mathrm{YKL}-40$ and CA125 levels were significantly higher $(P<$ 0.01) in the former.

The ROC curves of YKL-40 and CA125 were constructed for EOC patients versus controls. As a diagnostic tool for EOC, the approximate area under the ROC curve of YKL-40 was $0.975(95 \% \mathrm{Cl}=0.945$ to 1.004$)$ compared to $0.902(95 \% \mathrm{Cl}=0.839$ to 0.965$)$ for CA125 ( $P=0.0192$; Figure 1). According to the ROC curve, when the cut-off value of YKL-40 was $121.5 \mathrm{ng} / \mathrm{mL}, \mathrm{YKL}-40$ has a sensitivity of $92.9 \%$, a specificity of $94.4 \%$, a positive predictive value of $95.1 \%$, and an accuracy of $93.6 \%$, respectively, for the detection of EOC patients. At the value of $35 \mathrm{U} / \mathrm{mL}, \mathrm{CA} 125$ had a sensitivity of $73.8 \%$, a specificity of $86.1 \%$, a positive predictive value of $86.1 \%$, and an accuracy of $79.5 \%$. The sensitivity of YKL-40 was significantly better than that of CA125 (92.9 vs $73.8 \%, P=0.008)$. When YKL-40 and CA125 were tested in parallel, the sensitivity was increased to $98.2 \%$, while the specificity was slightly decreased from 86.1 to $81.3 \%$. But when they were tested in series, the specificity was increased to $99.2 \%$, while the sensitivity was decreased to $68.5 \%$.

In the EOC group, 39 of 42 (93\%) patients had elevated

Table 2. Serum YKL-40 and CA125 values of the subgroups studied.

\begin{tabular}{lcccc}
\hline Subgroup & $\begin{array}{c}\text { Healthy women } \\
(\mathrm{N}=46)\end{array}$ & $\begin{array}{c}\text { BGD } \\
(\mathrm{N}=42)\end{array}$ & $\begin{array}{c}\text { BEOT } \\
(\mathrm{N}=7)\end{array}$ & $\begin{array}{c}\text { EOC } \\
(\mathrm{N}=42)\end{array}$ \\
\hline $\begin{array}{l}\text { YKL-40 value }(\mathrm{ng} / \mathrm{mL}) \\
\text { Mean }\end{array}$ & 61.6 & 50.1 & $205.1^{*}$ & $220.1^{*}$ \\
Median & 60.5 & 44.3 & 201.0 & 192.7 \\
Range & $4.6-142.8$ & $8.8-182.6$ & $123.3-256.8$ & $71.9-495.6$ \\
Mean $\pm \mathrm{SD}$ & $61.6 \pm 48.4$ & $50.1 \pm 41.2$ & $205.1 \pm 43.8$ & $220.1 \pm 94.1$ \\
CA125 value $(\mathrm{U} / \mathrm{mL})$ & & & & \\
Mean & 13.4 & 28.5 & $243.8^{+}$ & $524.9^{+}$ \\
Median & 11.5 & 15.9 & 134.8 & 306.5 \\
Range & $5-32$ & $5.1-100.8$ & $9.9-600$ & $13.4-5000$ \\
Mean $\pm \mathrm{SD}$ & $13.4 \pm 7.6$ & $28.5 \pm 29.6$ & $243.8 \pm 238.6$ & $524.9 \pm 972.5$ \\
\hline
\end{tabular}

$\mathrm{BGD}=$ benign gynecological disease; $\mathrm{BEOT}=$ borderline epithelial ovarian tumor; EOC = epithelial ovarian cancer. ${ }^{*} \mathrm{P}<0.05$ for BEOT or EOC compared to healthy women or BGD (YKL-40; LSD test). ${ }^{+} \mathrm{P}<0.05$ for BEOT or ECO compared to healthy women or BGD (CA125; LSD test).

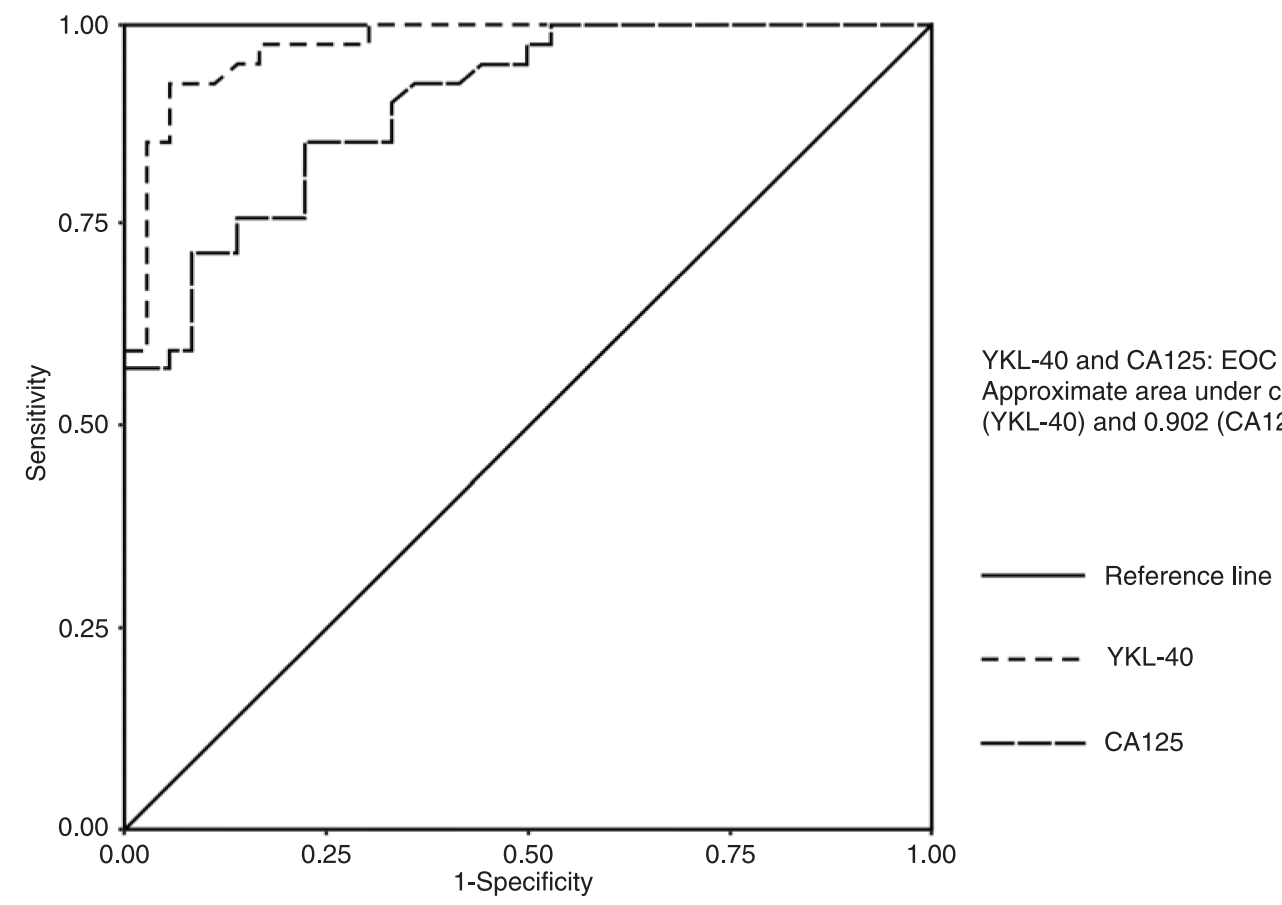

Figure 1. Receiver operating characteristic (ROC) curves of YKL-40 and CA125 for patients with epithelial ovarian cancer (EOC). $\mathrm{P}<0.05$ for approximate area under the ROC curve for YKL-40 compared to CA125 (LSD test). 
serum YKL-40 levels ( $\geq 121.5 \mathrm{ng} / \mathrm{mL})$, while 31 of $42(74 \%)$ patients had elevated serum CA125 levels ( $\geq 35 \mathrm{U} / \mathrm{mL}$; $93 \mathrm{vs}$ $74 \% ; P=0.008)$. Of 14 early-stage EOC patients, $11(79 \%)$ had elevated $\mathrm{YKL}-40$ levels but only six (43\%) had elevated CA125 levels $(P=0.120)$. Among 10 EOC patients whose serum CA125 levels were normal $(<35 \mathrm{U} / \mathrm{mL}), 7(70 \%)$ had elevated YKL-40 levels. In the borderline epithelial ovarian tumor subgroup, the serum YKL-40 levels were elevated in all patients $(7 / 7)$ but the CA125 levels were elevated in six patients $(6 / 7)$.

There were five subtypes of histology, including papserous, clear cell, endometrioid, mixed gland, and others in 42 EOC patients (Table 1). YKL-40 and CA125 were both elevated in all (100\%) 18 patients with serous carcinoma. Among 24 patients with non-serous malignant tumors, YKL40 and CA125 were elevated in $21(88 \%)$ and $13(54 \%)(P$ $=0.011$ ), respectively.

The preoperative $\mathrm{YKL}-40$ value increased with increasing FIGO stage of EOC. Patients with advanced-stage
EOC had 1.42 times higher preoperative YKL-40 values than those of patients with early-stage EOC. There was a linear trend in YKL-40 elevation when YKL-40 levels were compared with FIGO stage I to IV by the Spearman rank correlation test $(r=0.297, \mathrm{P}=0.029$; Figure 2A).

Preoperative $\mathrm{YKL}-40$ values increased with increasing histological grade. Elevated serum YKL-40 levels ( $\geq 121.5$ $\mathrm{ng} / \mathrm{mL}$ ) were present in $67 \%(2 / 3)$ of patients with histological grade 1 , as opposed to $75 \%(6 / 8)$ and $100 \%$ (31/31) of patients with histological grades 2 and 3 , respectively. There was a linear trend in YKL-40 elevation when YKL-40 levels were compared to histological grade using Spearman's rank correlation $(r=0.303, \mathrm{P}=0.05$; Figure $2 \mathrm{~B})$. However, there was no linear trend between $\mathrm{YKL}-40$ and histology $(P=N S$; Figure 2C). Also, no linear trend was observed between YKL-40 and performance status, age or extension of debulking surgery (data not shown).

Five EOC patients were lost to follow-up and 12 $(32.43 \%)$ of 37 patients with EOC died. Univariate Cox
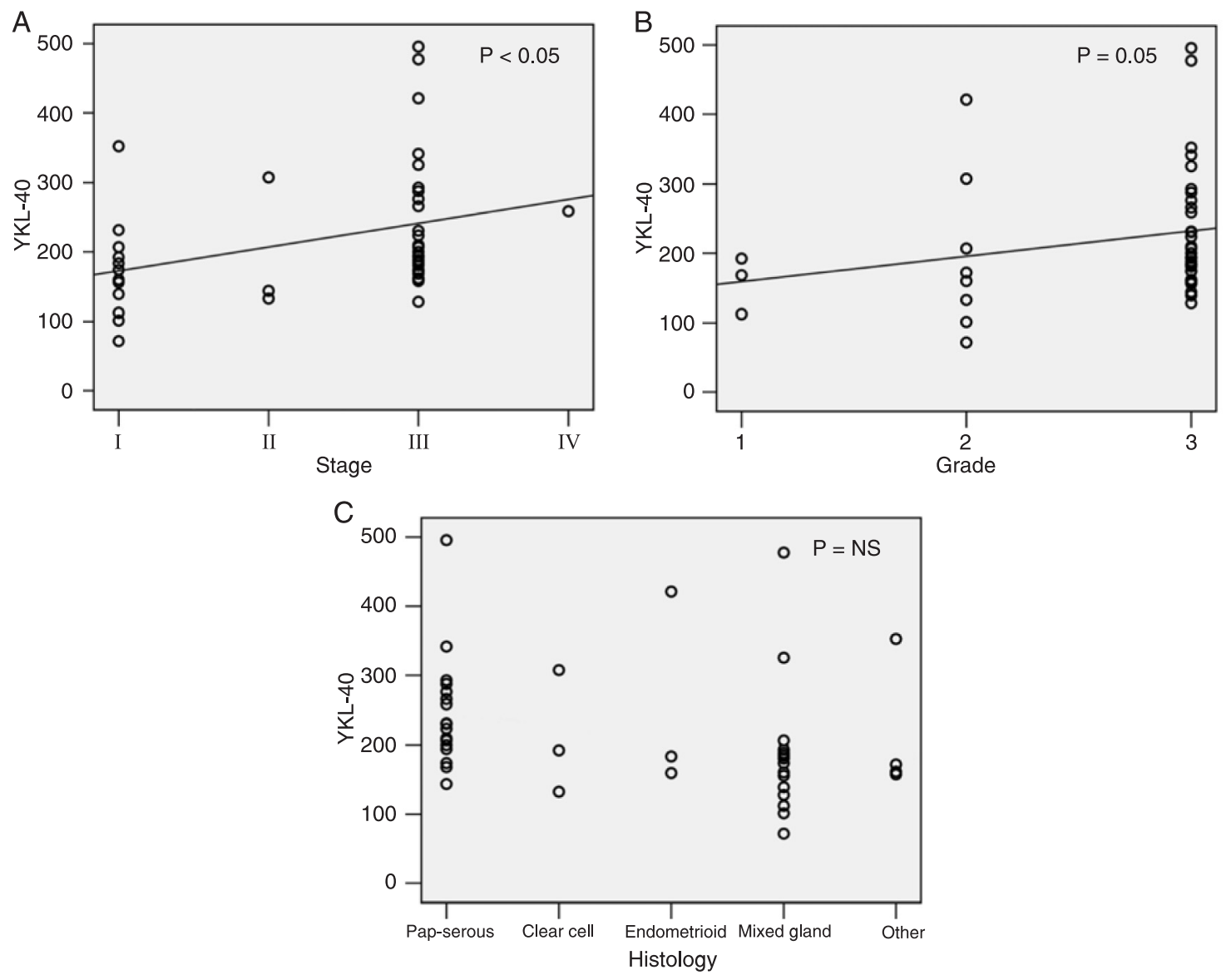

Figure 2. Association of $Y K L-40$ with stage $(A ; P<0.05)$, grade $(B ; P=0.05)$, and histology $(C ; P=N S)$ (Spearman rankcorrelation coefficient test). Each dot represents the serum YKL-40 value of 1 patient with epithelial ovarian cancer. The regression line is shown in Panels $A$ and $B$. 
analysis of serum YKL-40 showed no significant association with overall survival $(P=0.171)$. Thirty-four of $37 \mathrm{EOC}$ patients had elevated serum YKL-40 levels (>121.5 $\mathrm{ng} / \mathrm{mL}$ ) and only 3 had normal serum YKL-40 levels. All of the patients who died had elevated YKL-40. The Kaplan-Meier survival estimates stratified by normal serum YKL-40 vs elevated serum YKL-40 are illustrated in Figure 3. Performing a multivariate Cox analysis including serum YKL-40, FIGO stage, serum CA-125, histologic grade, performance status, age, and extension of debulking surgery showed that all the parameters had no independent prognostic value in EOC patients.

\section{Discussion}

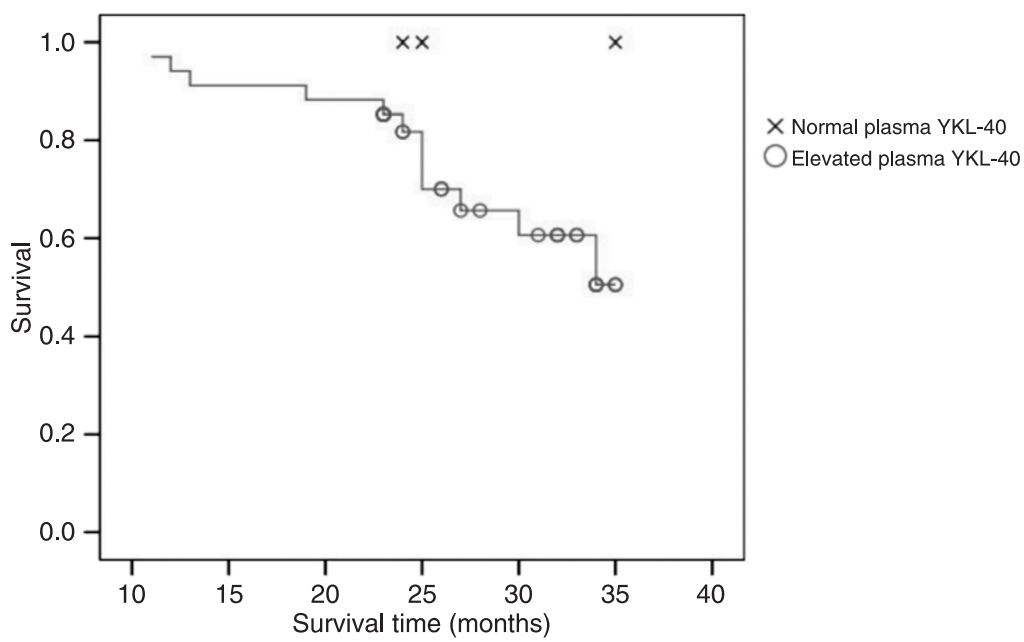

Figure 3. Kaplan-Meier survival estimates for patients with epithelial ovarian cancer according to normal and elevated serum levels of YKL-40.

The high mortality of ovarian cancer is primarily caused by the absence of efficient methods of preoperative diagnosis and recurrence detection. Tumor biomarkers have been regarded as effective and convenient methods for detecting ovarian cancer before surgery.

CA125 is a widely used biomarker for detecting patients with EOC, the leading histology in all malignant ovarian tumors (14). But sensitivity and specificity for the diagnosis of patients with ovarian cancer are still unsatisfactory (16). Only 40 to $50 \%$ patients with stage I ovarian tumors can be detected on the basis of elevated serum CA125 (17). Even when CA125 is used in combination with a range of various biomarkers, sensitivity ranges from 60.8 to $82.1 \%$ (18).

Although in vivo proof is yet to be obtained, it has been suggested that YKL-40 may have an important effect on the proliferation and differentiation of cancer cells, and on the prevention of cell apoptosis, and may play a role in extracellular tissue remodeling, and stimulate angiogenesis and fibroblasts surrounding the tumor $(1,2)$. Clinical studies of patients with different types of solid tumors have indicated that YKL-40 values are remarkably elevated in cancer patients compared to healthy women $(9-11,16,19)$. Moreover, YKL-40 has been regarded as a "biomarker" or "possible cancer marker" for detecting some malignant tumors $(7,9,16,19,20)$.

It has been reported that YKL-40 is strongly dependent on handling procedures, although freezing and thawing repeated eight times had no significant effect on the YKL-40 concentrations measured $(6,21)$. All blood samples must be processed to obtain plasma within $8 \mathrm{~h}$ at room temperature or to obtain serum within less than $3 \mathrm{~h}$ at room temperature $(6,21)$. In the present study, all blood samples had been processed to obtain serum within $3 \mathrm{~h}$ before centrifugation. Each serum sample was subdivided into several smaller volumes and stored at $-80^{\circ} \mathrm{C}$ until testing, avoiding repetitive freezing and thawing. Thus, the YKL-40 values measured in our research were reliable and repeatable.

In the present study, we analyzed and compared the diagnostic parameters of $\mathrm{YKL}-40$ and CA125 in order to assess the potency of YKL-40 alone or in combination with CA125 for the diagnosis of ovarian cancer. YKL-40 and CA125 levels were both significantly higher in the malignant group than in the control group, indicating that YKL-40 may act as a biomarker to distinguish patients with ovarian cancer from patients with no malignancy.

The approximate area under the ROC curve evaluating serum YKL-40 for the detection of ovarian cancer against controls was 0.975 compared to 0.902 for CA125 ( $P=$ $0.0192)$. The sensitivity of $Y K L-40$ was significantly better than that of CA125, indicating that YKL-40 may be a valuable biomarker for the diagnosis of ovarian cancer. When YKL-40 and CA125 were tested in parallel, sensitivity increased from 73.8 to $98.2 \%$, while specificity was slightly decreased from 86.1 to $81.3 \%$. Thus, the combination of YKL-40 and CA125 seems to be preferable for the diagnosis of ovarian cancer. In 24 patients with non-serous histology for EOC, YKL-40 levels were higher than CA125 levels. These results indicate that YKL-40 may be more sensitive than CA125 in detecting EOC and that more EOC patients could be detected if YKL-40 and CA125 levels were tested in parallel.

Currently, there is a tendency towards a new grading system for ovarian cancer, which has been approved by the International Society of Gynecologic Pathologists. In this new system, a 2-tier system has been proposed in which serous carcinomas are subdivided into low and high grades (22), while endometrioid carcinomas are still subdivided into grades 1-3. Some special histological subtypes, including clear cell, transitional cell and undifferentiated carcinomas, are all classified into high grade. Because of the limited number of cases of every histological subtype, in which the 
histological grade of EOC was subdivided into grades 1-3, the traditional FIGO grading system was used to classify our ovarian cancer patients.

In order to identify the possible mechanism of $\mathrm{YKL}-40$ as a biomarker for the detection of EOC, the correlations between YKL-40 value and FIGO stage, histological grade, and histology were tested. In our study, preoperative serum levels of YKL-40 increased linearly with increasing FIGO stage and histological EOC grade. These results partly explained why YKL-40 may be a biomarker for the detection of EOC. However, the associations between YKL-40 and stage and histological grade, although significant, were rather weak because of the traditional FIGO grading system, limited number of each subtype and unbalanced distribution of case numbers.

Some reports have indicated that YKL-40 is associated with the survival of ovarian cancer patients, being even regarded as the independent prognostic marker of overall survival $(11,23)$. However, our results indicated

\section{References}

1. Renkema GH, Boot RG, Muijsers AO, Donker-Koopman WE, Aerts JM. Purification and characterization of human chitotriosidase, a novel member of the chitinase family of proteins. J Biol Chem 1995; 270: 2198-2202.

2. Johansen JS, Williamson MK, Rice JS, Price PA. Identification of proteins secreted by human osteoblastic cells in culture. J Bone Miner Res 1992; 7: 501-512.

3. Johansen JS, Jensen HS, Price PA. A new biochemical marker for joint injury. Analysis of YKL-40 in serum and synovial fluid. Br J Rheumatol 1993; 32: 949-955.

4. Vind I, Johansen JS, Price PA, Munkholm P. Serum YKL-40, a potential new marker of disease activity in patients with inflammatory bowel disease. Scand J Gastroenterol 2003; 38: 599-605.

5. Johansen JS, Jensen BV, Roslind A, Nielsen D, Price PA. Serum YKL-40, a new prognostic biomarker in cancer patients? Cancer Epidemiol Biomarkers Prev 2006; 15: 194202.

6. Cintin C, Johansen JS, Christensen IJ, Price PA, Sorensen $\mathrm{S}$, Nielsen HJ. Serum YKL-40 and colorectal cancer. $\mathrm{Br} J$ Cancer 1999; 79: 1494-1499.

7. Johansen JS, Cintin C, Jorgensen M, Kamby C, Price PA. Serum YKL-40: a new potential marker of prognosis and location of metastases of patients with recurrent breast cancer. Eur J Cancer 1995; 31A: 1437-1442.

8. Johansen JS, Roslind A, Palle C, Christensen IJ, Nielsen HJ, Price PA, et al. Serum YKL-40 levels in patients with cervical cancer are elevated compared to patients with cervical intraepithelial neoplasia and healthy controls. $J$ Clin Oncol 2006; 24 (Suppl 1): 267s.

9. Diefenbach CS, Shah Z, lasonos A, Barakat RR, Levine DA, Aghajanian C, et al. Preoperative serum YKL-40 is a marker for detection and prognosis of endometrial cancer. Gynecol Oncol 2007; 104: 435-442.

10. Dupont J, Tanwar MK, Thaler HT, Fleisher M, Kauff N, Hensley $\mathrm{ML}$, et al. Early detection and prognosis of ovarian cancer the opposite result, possibly due to several reasons. First, only 3 EOC patients had normal YKL-40 levels and they were all alive at the end of follow-up. A small number of deaths of patients with normal YKL-40 was the major reason. In addition, follow-up time was not long enough and some patients might die at later times, a fact that makes the prognostic value of YKL-40 less attractive. Finally, the limited number of patients may have been inadequate to clarify the prognostic value of YKL-40.

Our results have shown the advantages of YKL-40 alone and in combination with CA125 as biomarkers for the detection of ovarian cancer. However, a longer follow-up time and reevaluation are needed to confirm the prognostic role of $\mathrm{YKL}-40$ in EOC patients.

\section{Acknowledgments}

Financial support was supplied by Professor Jiawen Zhang and Sichuan University.

using serum YKL-40. J Clin Oncol 2004; 22: 3330-3339.

11. Hogdall EV, Ringsholt M, Hogdall CK, Christensen IJ, Johansen JS, Kjaer SK, et al. YKL-40 tissue expression and plasma levels in patients with ovarian cancer. BMC Cancer 2009; 9: 8.

12. Chetrit A, Hirsh-Yechezkel G, Ben-David Y, Lubin F, Friedman E, Sadetzki S. Effect of BRCA1/2 mutations on longterm survival of patients with invasive ovarian cancer: The National Israeli Study of Ovarian Cancer. J Clin Oncol 2008; 26: 20-25.

13. Ozols RF, Rubin SC, Thomas GM, Robboy SJ. Epithelial ovarian cancer. In: Hoskins WJ, Perez CA, Young RC, Barakat RR, Markman M, Randall ME (Editors), Principles and practice of gynecologic oncology. 4th edn. Philadelphia: Lippincott Williams \& Wilkins; 2005. p 895-987.

14. Terry KL, Sluss PM, Skates SJ, Mok SC, Ye B, Vitonis AF, et al. Blood and urine markers for ovarian cancer: a comprehensive review. Dis Markers 2004; 20: 53-70.

15. Dehn H, Hogdall EV, Johansen JS, Jorgensen M, Price PA, Engelholm SA, et al. Plasma YKL-40, as a prognostic tumor marker in recurrent ovarian cancer. Acta Obstet Gynecol Scand 2003; 82: 287-293.

16. Malkasian GD Jr, Knapp RC, Lavin PT, Zurawski VR Jr, Podratz KC, Stanhope CR, et al. Preoperative evaluation of serum CA 125 levels in premenopausal and postmenopausal patients with pelvic masses: discrimination of benign from malignant disease. Am J Obstet Gynecol 1988; 159: 341-346.

17. Bast RC Jr, Xu FJ, Yu YH, Barnhill S, Zhang Z, Mills GB. CA 125: the past and the future. Int J Biol Markers 1998; 13: 179-187.

18. Moore RG, Brown AK, Miller MC, Skates S, Allard WJ, Verch $\mathrm{T}$, et al. The use of multiple novel tumor biomarkers for the detection of ovarian carcinoma in patients with a pelvic mass. Gynecol Oncol 2008; 108: 402-408.

19. Johansen JS, Hansen U, Christensen IBJ, Garbarsch C, 
Price PA, Nielsen HJ. YKL-40 protein expression in cancer cells and macrophages in colorectal cancer. $J$ Clin Oncol 2005; 23 (Suppl 1): 300s.

20. Johansen JS, Christensen IJ, Riisbro R, Greenall M, Han C, Price PA, et al. High serum YKL-40 levels in patients with primary breast cancer is related to short recurrence free survival. Breast Cancer Res Treat 2003; 80: 15-21.

21. Hogdall EV, Johansen JS, Kjaer SK, Price PA, Blaakjaer J, Hogdall CK. Stability of YKL-40 concentration in blood samples. Scand J Clin Lab Invest 2000; 60: 247-251.

22. Vang R, Shih I, Kurman RJ. Ovarian low-grade and highgrade serous carcinoma: pathogenesis, clinicopathologic and molecular biologic features, and diagnostic problems. Adv Anat Pathol 2009; 16: 267-282.

23. Hogdall EV, Johansen JS, Kjaer SK, Price PA, Christensen $\mathrm{L}$, Blaakaer J, et al. High plasma YKL-40 level in patients with ovarian cancer stage III is related to shorter survival. Oncol Rep 2003; 10: 1535-1538. 